\title{
Monte Carlo-Based Indoor RFID Positioning with Dual-Antenna Joint Rectification
}

\author{
Yonghui Tao ${ }^{1}$, Lujie $\mathrm{Wu}^{2}$, Johan Sidén ${ }^{3} \mathbb{1}$ and Gang Wang ${ }^{2, *}$ \\ 1 Department of Electronic and Information Engineering, Jinling Institute of Technology, \\ Nanjing 211169, China; yhtao87@jit.edu.cn \\ 2 Department of Electronic Engineering and Information Science, University of Science and Technology of \\ China, Hefei 230027, China; wljie@mail.ustc.edu.cn \\ 3 Department of Electronics Design, Mid Sweden University, SE-851 70 Sundsvall, Sweden; \\ Johan.Siden@miun.se \\ * Correspondence: gwang01@ustc.edu.cn
}

Citation: Tao, Y.; Wu, L.; Sidén, J.; Wang, G. Monte Carlo-Based Indoor RFID Positioning with Dual-Antenna Joint Rectification. Electronics 2021, 10, 1548. https://doi.org/10.3390/ electronics10131548

Academic Editor: Dimitra

I. Kaklamani

Received: 27 April 2021

Accepted: 21 June 2021

Published: 25 June 2021

Publisher's Note: MDPI stays neutral with regard to jurisdictional claims in published maps and institutional affiliations.

Copyright: () 2021 by the authors. Licensee MDPI, Basel, Switzerland. This article is an open access article distributed under the terms and conditions of the Creative Commons Attribution (CC BY) license (https:// creativecommons.org/licenses/by/ $4.0 /)$.

\begin{abstract}
A novel Monte Carlo-based indoor radio-frequency identification (RFID) positioning scheme is proposed for dual-antenna RFID systems with the cooperation of dual-antenna joint rectification. By deploying reference passive RFID tags on the ground to establish an RFID tag-based map, indoor self-positioning of a moving platform carrying an RFID reader with two forward-looking antennas can be simply implemented by looking up the positions of responded RFID tags at each time step of movement, and estimating the platform position by using the proposed Monte Carlo-based algorithm. To improve the positioning accuracy of Monte Carlo-based positioning, each antenna channel, with its own footprint on the ground, may rectify its position estimation by using the tag position information interrogated by the other antenna channel. The algorithm for dual-antenna rectification is proposed. The performance of the proposed Monte Carlo-based self-positioning scheme is demonstrated by both simulation and experiment tests. Some factors in a practical indoorpositioning system, such as the reference tag distribution pattern, reader antenna footprint size, and footprint overlap, are discussed. Some guide rules for deploying the RFID indoor-positioning system are also reported.
\end{abstract}

Keywords: accuracy; antenna; indoor positioning; Monte Carlo; radio-frequency identification; ultra-high frequency

\section{Introduction}

Nowadays, indoor localization technology has been widely used in customer navigation in shopping malls, warehouse management, object localization and tracking in airports, etc. Based on prevailing wireless communication technology, including WiFi, Bluetooth, UWB, Radio Frequency Identification (RFID) Device, visible light communication, etc., several wireless position techniques have been proposed for indoor applications. In [1], an indoor wireless positioning scheme has been proposed based on WiFi fingerprints. In [2], a multimodal approach is proposed to enhance indoor localization with camera and WiFi signals. The positioning error can be controlled in $0.2 \mathrm{~m}$, under the case of enough WiFi access points and cameras. In [3], a fingerprinting localization algorithm with fuzzy logic type-2 using Bluetooth Low Energy (BLE) beacons is proposed. The localization error was $0.43 \mathrm{~m}$. In [4], a localization scheme using Visible Light Communication (VLC) is proposed, which achieves a positioning accuracy within $1-2 \mathrm{~cm}$. The limitation is that there must be a line-of-sight transmission path between the LED and the sensor.

In general, these positioning schemes have their own advantages and disadvantages. For some environments, such as warehouses, retail shops, libraries, etc., the positioning with passive RFID technology at ultra-high frequency (UHF) band represents a costeffective solution because the passive RFID tags are so cheap nowadays and can be easily 
deployed as reference tags for RFID-based indoor positioning, with almost no worries about the number of reference tags required.

By interrogating the specifically deployed passive RFID tags, recording tags response, and combing the positions of RFID tags, the position of RFID reader or mobile platform carrying RFID reader can be obtained in a unique manner [5]. Owing to the specific answer range of passive RFID tags, an RFID-based indoor positioning scheme may provide more accurate positioning results compared to other wireless positioning indoor schemes $[6,7]$. Since the RFID reader and tag are becoming cheaper and cheaper, the RFID-based positioning scheme shows great potentials in meeting the trend of RFID applications.

For RFID-based indoor wireless positioning, quite a few positioning algorithms and positioning schemes have been reported in recent years [8-12]. The most common RFIDbased indoor positioning scheme is constructed with an RFID reader on a moving platform connected to a single RFID reader antenna [8,9,12]. A general RFID indoor positioning system tries to range the distances between the reader antenna and RFID tags by using the received signal strength indicator (RSSI) $[13,14]$. However, the RSSI may be distorted by poor indoor wireless propagation to some extent due to the interference from different obstacles. In order to improve positioning accuracy, several statistical algorithms have been proposed by eliminating the interference or noise from the indoor environment [7,15-20]. One representative algorithm is based on the Kalman filter, which is proposed as an alternative for real-time positioning [15,18-20]. An Extended Kalman filter has also been proposed to suppress further the effects of environmental noise [21,22]. In general, all these positioning schemes are based on odometry, which improves the positioning accuracy by combining the tags' position information.

In order to improve the positioning accuracy further, RFID-indoor positioning schemes using multiple RFID antennas have been proposed [23-30]. By using two or more RFID reader antennas for positioning, more tag information can be collected so that the positioning accuracy can be improved if proper positioning algorithms are used. Multiple directional and steerable antennas are considered to increase accuracy in [24]. In [25], the direction of arrival of tag signals gathered from dual-directional antennas is used to improve the position accuracy. Multiple antennas that were situated to overlap the recognition areas to read more tags simultaneously are used to improve the accuracy of position estimation in [26,27]. In [28], reference tags are introduced to the RFID system with two antennas, and the positioning is performed essentially with a particle filter-based method by updating the particles algorithm in a timely and accurate manner. In [30], an indoor localization method based on both the angle of arrival and phase difference of arrival using is proposed for an RFID system with an array antenna and virtual stations.

The Monte Carlo algorithm provides a unique positioning scheme for RFID indoor application. The Monte Carlo algorithm has found applications in wireless positioning [31,32], and improved Monte Carlo algorithm in RFID-based localization [33,34]. The main idea of the Monte-Carlo based algorithm is to use substantial sample points from the state space to approximate the location distribution estimation. With the sample points referred to as the particles, the integral problem in Bayesian iteration is transformed into the summation problem. For RFID-indoor positioning, the positions of specifically deployed passive RFID tags that respond to the interrogation of RFID reader at consequent moving steps will make the RFID-based positioning, which is quite reasonable for the indoor environment with interference from different obstacles, even for scenarios of a dynamic indoor environment. In this paper, we will focus on the Monte Carlo-based scheme for positioning a moving platform carrying one RFID reader with two or more reader antennas.

The paper is organized as follows. Section 2 describes the proposed Monte Carlo-based positioning scheme for a dual-antenna RFID indoor positioning system. The rectification of each reader antenna channel for joint positioning is provided. In Section 3, the performance of the proposed Monte Carlo-based positioning scheme is demonstrated by numerical simulations. In Section 4, experimental results are reported to demonstrate the proposed indoor positioning scheme. In Section 5, some practical factors that affect the positioning 
accuracy are further analyzed, which provides some guide rules in deploying the proposed Monte Carlo-based indoor RFID positioning scheme.

\section{Monte Carlo-Based RFID Positioning with Dual-Antenna Joint Rectification}

\subsection{Dual-Antenna RFID Indoor Positioning System}

As shown in Figure 1, suppose that a moving vehicle (a fork truck, for example) with an RFID system of two RFID reader antennas is to be positioned in an indoor environment. Each RFID reader antenna has a circular footprint of radius $R$ on the ground and can interrogate several RFID tags in the footprint.

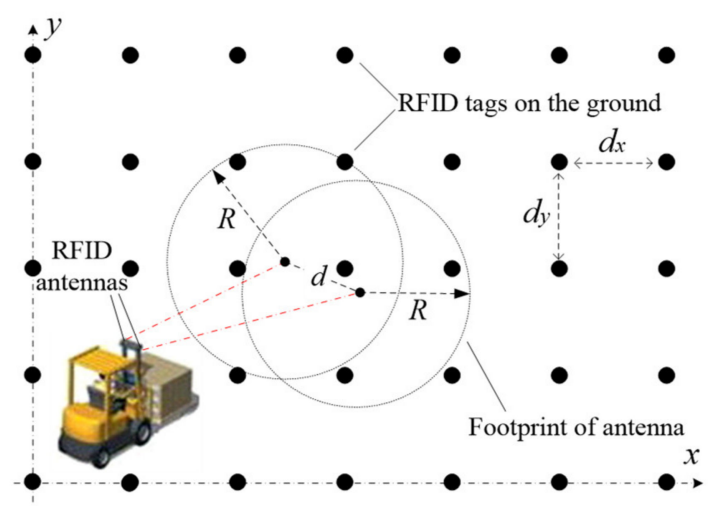

Figure 1. Indoor positioning of dual-antenna RFID system.

For positioning, UHF passive RFID tags are deployed on the ground in a designated pattern that two neighboring tags have intervals of $d_{x}$ in the $x$-direction and $d_{y}$ in the $y$ direction. The ID information of the RFID tag is bounded together with its coordinate positions on the ground so that an RFID-based electronic map can be defined in terms of tags' IDs and positions.

In the practical deployment of the RFID positioning system, the two RFID reader antennas can be set in a forward-looking pattern. The geometry of a dual-antenna RFID positioning system is shown in Figure 2a. For the adjustment of footprints on the ground in our demon system, several rotary joints are designed to adjust the footprints. The diagram of the dual-antenna RFID-based indoor positioning is shown in Figure $2 b$.

From the geometry in Figure 2, the vehicle's position can be determined by knowing the positions of the two centers of RFID antennas' footprints on the ground. Theoretically, the position of each footprint center can be acquired by using the Monte-Carlo positioning algorithm if following the positioning scheme for RFID systems with a single reader antenna. Since there are two antennas connected to a reader and the information acquired by the two antenna channels may help each other in positioning, we will see how to merge the information from the two antenna channels.

Suppose two footprint centers on the ground are positioned at $\left(x_{1}, y_{1}\right)$ and $\left(x_{2}, y_{2}\right)$, phase centers of the two RFID antennas are projected on the ground at $\left(x_{10}, y_{10}\right)$ and $\left(x_{20}, y_{20}\right)$; the geometry in Figure 2 gives

$$
\begin{gathered}
x_{10}=x_{1} \pm \frac{(h \tan (\theta))^{2}\left|y_{2}-y_{1}\right|}{d}, \\
y_{10}=y_{1} \pm \frac{(h \tan (\theta))^{2}}{d\left(x_{2}-x_{1}\right)}, \\
x_{20}=x_{2} \pm \frac{(h \tan (\theta))^{2}\left|y_{2}-y_{1}\right|}{d},
\end{gathered}
$$




$$
y_{20}=y_{2} \pm \frac{(h \tan (\theta))^{2}}{d\left(x_{2}-x_{1}\right)}
$$

where $h$ is the height of the antenna above the ground, $d$ is the interval between the two footprint centers, and $\theta$ is the tilt angle to set the beam of the RFID antenna. The vehicle position $\left(x_{0}, y_{0}\right)$ can be acquired by

$$
x_{0}=\frac{x_{10}+x_{20}}{2}, y_{0}=\frac{y_{10}+y_{20}}{2} .
$$

Therefore, to position the two footprint centers is the key task.

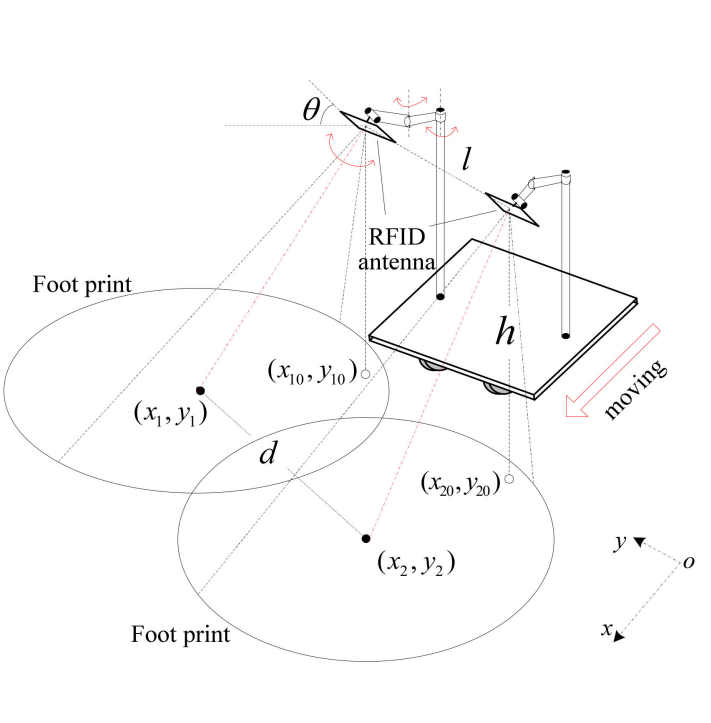

(a)

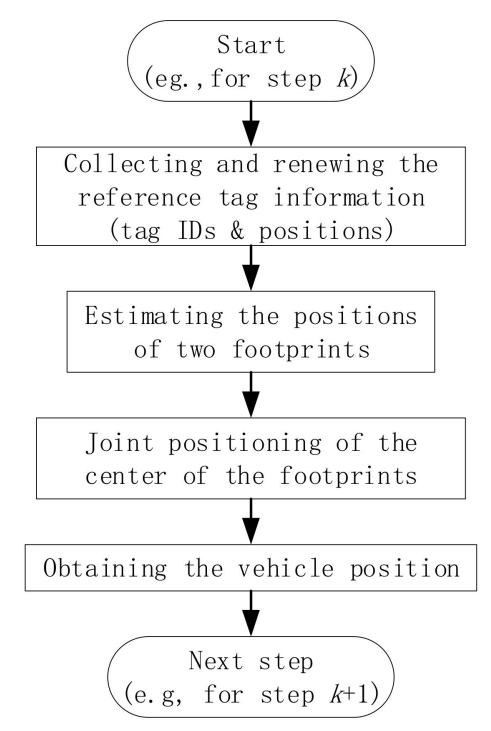

(b)

Figure 2. Geometry of the dual-antenna RFID positioning system (a) and diagram of the proposed dual-antenna RFID positioning scheme (b).

It should be noted that a circularly polarized RFID reader antenna can be implemented in practice so that possible polarization mismatch between the reader antennas and RFID tags during the move of the vehicle can be avoided, and all tags in footprint can be interrogated. The tilt angle of the reader antenna should be carefully adjusted so that overlapping of the two footprints on the ground can be joined or separated as desired.

In practice, the footprint of the RFID reader antenna on the ground can be obtained by recording the response of a reference RFID tag when moving on the ground.

\subsection{Dual-Antenna RFID Indoor Positioning System}

The movement of a vehicle can be indicated by its position at different time steps. At each time step, the position of the footprint center can be estimated by the Monte Carlo algorithm by using the positions of RFID tags that are within the RFID antenna's footprint. Therefore, the Monte Carlo-based positioning is a step-by-step scheme.

The Monte Carlo positioning algorithm is implemented by two stages: prediction stage and updating stage. At the prediction stage, data of sampling particles (i.e., possible footprint center position) at the present time step are estimated from particles at the previous time step. At the updating stage, particles are updated, and the position of a footprint center is obtained.

\subsubsection{Prediction Stage}

At the end of time step $(k+1)$, the intelligent control center on the vehicle will predict the next position of a footprint center for step $k$. Monte Carlo prediction is made in 
a probability manner based on positions of the RFID tags which answer the RFID reader's interrogation.

Suppose an RFID reader antenna can detect $n$ tags on the ground at time step $k$, which have coordinates of $\left(x_{i}^{k}, y_{i}^{k}\right)(i=1,2, \ldots, n)$, respectively. The preliminary position of a footprint center for step $k$ can be estimated as

$$
\left(a x_{k}^{\prime}, a y_{k}^{\prime}\right)=\left(\frac{1}{n} \sum_{i=1}^{n} x_{i}^{k}, \frac{1}{n} \sum_{i=1}^{n} y_{i}^{k}\right) .
$$

In general, the preliminary position may deviate from the real footprint center. Thus a probability density function can be defined by using $N_{0}$ sampling particles to yield a more precise footprint center position. Among the $N_{0}$ sampling particles, the $i$-th particle is composed of a hypothesized footprint center position $s_{i}^{k}=\left(s x_{i}, s y_{i}\right)$ with a probability weight of $w_{i}$. The particle position $s_{i}^{k}=\left(s x_{i}, s y_{i}\right)$ is thus located randomly in the circle of the radius $r$ centered at $\left(a x_{k}^{\prime}, a y_{k}^{\prime}\right)$ with a certain probability (which will be evaluated in the next subsection). Therefore, the sampling particle at time $k$ is given by

$$
S_{k}=\left\{\left(s_{k}^{i}, w_{k}^{i}\right) \mid i=1,2, \cdots, N_{0}\right\} .
$$

In theory, each sampling particle should satisfy the system condition, i.e., the footprint defined by the particle should cover at least three tags identified by the reader. Otherwise, the particle position will be replaced by the average of coordinates of the two adjacent particles which satisfy the system condition. This is very important, especially in case that the RFID positioning system with one RFID reader antenna cannot interrogate enough RFID tags (less than three) on the ground. By choosing two adjacent particles, averaging their coordinates, and complementing the missing particle position, the prediction sequence can proceed smoothly.

\subsubsection{Dynamic Updating Stage}

At the time step $k+1$, the vehicle will move to a new position and detect some different RFID tags, and the sampling particles $S_{k}$ defined at time step $k$ will update to $S_{k+1}$. In the updating to $S_{k+1}$, each particle should meet the system condition.

Supposing the estimation takes a Gaussian probability model, we may evaluate the weight $w_{i}$ for those available particles as

$$
w_{k}^{i}=p\left(D_{i}^{k} \mid P_{k-1}\right)=\frac{1}{\sigma \sqrt{2 \pi}} e^{\frac{\left(D_{i}^{k}-O_{k-1}\right)^{2}}{2 \sigma^{2}}},
$$

where $D_{i}^{k}$ is the distance between the $i$-th particle at time step $k$ and the footprint center at time step $k-1, P_{k-1}$ is the position of the footprint center at time step $k-1, Q_{k-1}$ is the distance between the footprint centers at time step $k-1$ and at time step $k$, and $\sigma$ is the variance of the measured value of $D_{i}^{k}$.

By normalizing the weights of the particle in set $S_{k}$, we may evaluate the position of footprint center at time $k$ as

$$
l_{k}=\sum_{i=1}^{N_{0}} s_{k}^{i} \bullet w_{k}^{i} \quad(k=2,3 \cdots \cdots N),
$$

where $N$ is the number of positioning steps. By repeating the above two phases recursively for every step, a full trace of the moving vehicle can be estimated and provided.

The Monte Carlo positioning algorithms can be implemented, shown in Algorithm 1 


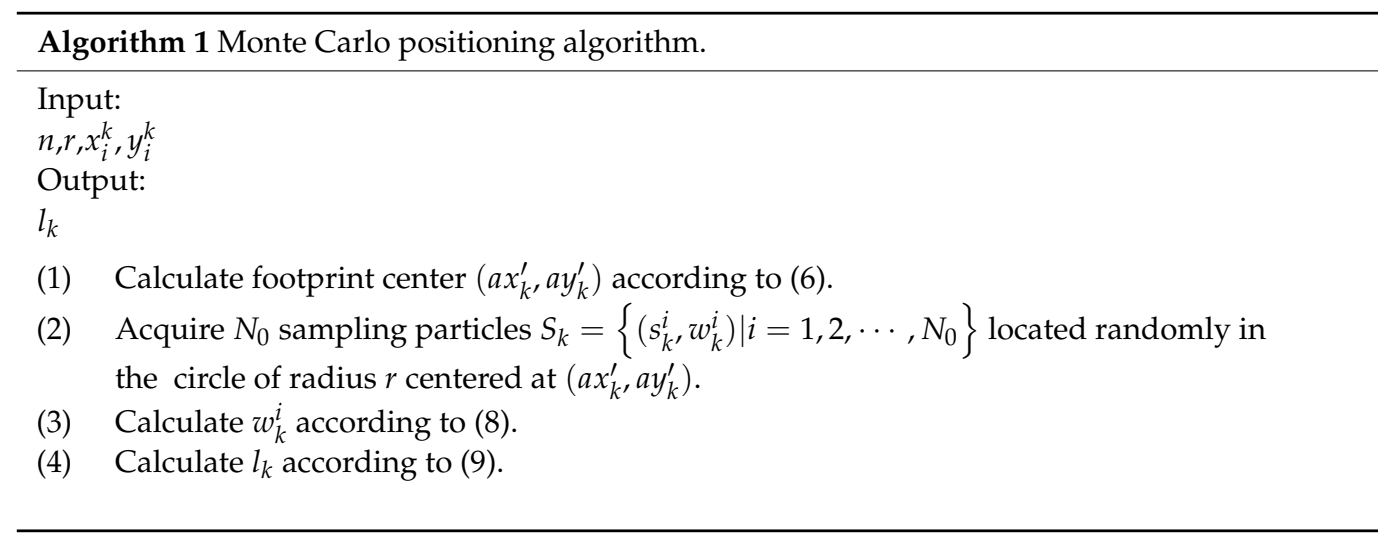

\subsection{Joint Rectification from the Two Footprints}

In Monte Carlo-based positioning, the accuracy of positioning is closely related to the number of tags that can be detected within the antenna's footprint. The positioning accuracy increases with the number of reference tags detected. For reliable positioning, two antennas in the RFID system will benefit the positioning.

For a vehicle moving in practical indoor environments, it is most likely that at some positions, RFID tags detected by a single antenna are not enough to guarantee positioning precision. For the dual-antenna RFID system, an advantage over the one-antenna RFID system can be expected because positioning based on tags detected by one antenna can be rectified or improved by tags detected by the other antenna.

According to the number of tags detected by the two RFID antennas (\#1 and \#2), typical rectification schemes can be proposed as follows.

\subsubsection{Both Antennas Detect Enough Tags}

For this scenario, both reader antenna \#1 and \#2 detect enough tags (at least three), so that the positioning for any of the RFID reader antennas is reliable. However, the position information of tags in one antenna's footprint can be lent to improve the precision of positioning of the other antenna, which leads to a joint positioning.

The benefits of joint positioning by using two RFID reader antennas can be acquired in different manners. Here, we give the most straightforward implementation. As shown in Figure 3, suppose $l$ is the distance between the two real footprint centers, and $l^{\prime}$ is the distance between the two footprint centers estimated by using the Monte Carlo algorithm. The joint position algorithm is as follows (shown in Algorithm 2):

(i) Extend line $l^{\prime}$ to intersect with two potential footprint center zones to acquire four intersections $\left(b_{11}, b_{12}, b_{21}, b_{22}\right)$, as depicted in Figure $4 \mathrm{a}$;

(ii) Connect the midpoints of line $b_{11} b_{12}$ and $b_{21} b_{22}$ to obtain a line $b_{1} b_{2}$, and the mobile vehicle position can be estimated as the midpoint $o_{k}^{\prime}$ of $b_{1} b_{2}$, as shown in Figure $4 \mathrm{~b}$;

(iii) Draw a circle of radius $l / 2$ centered at $o_{k}^{\prime}$, then two circular segments $c_{11} c_{12}$ and $c_{21} c_{22}$ can be founded in two potential footprint center areas, as shown in Figure $4 \mathrm{c}$

(iv) The midpoints of $c_{11} c_{12}$ and $c_{21} c_{22}$ gives the footprint centers $\left(x_{10}, y_{10}\right)$ and $\left(x_{20}, y_{20}\right)$; of antenna \#1 and \#2, respectively, as shown in Figure $4 \mathrm{~d}$.

Once the positions of the two antennas are determined, the vehicle position $\left(x_{0}, y_{0}\right)$ can be acquired by (5), shown in Algorithm 2. Below is a simple implementation: 

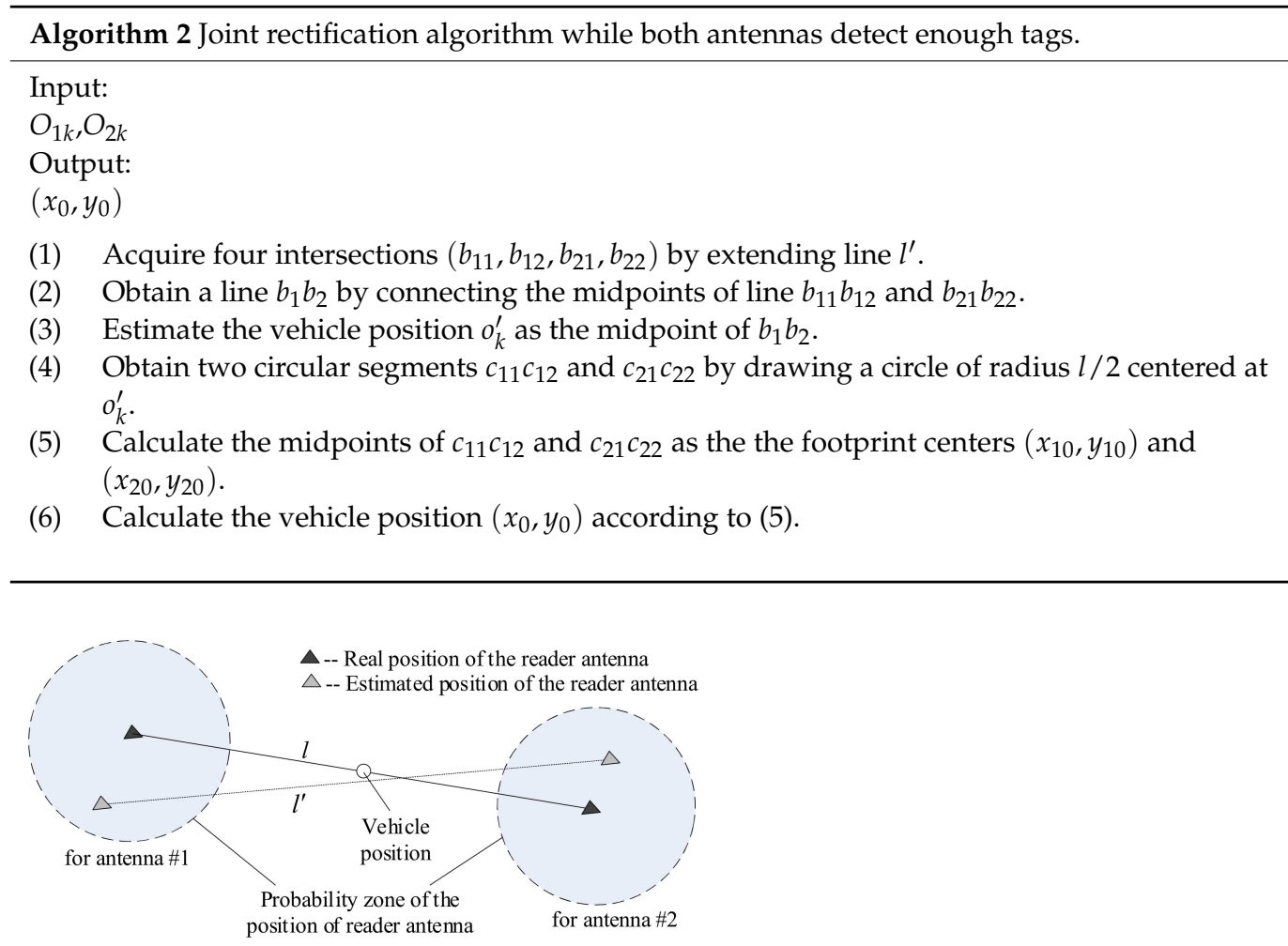

Figure 3. Scenario for positioning when both reader antennas can detect enough tags.

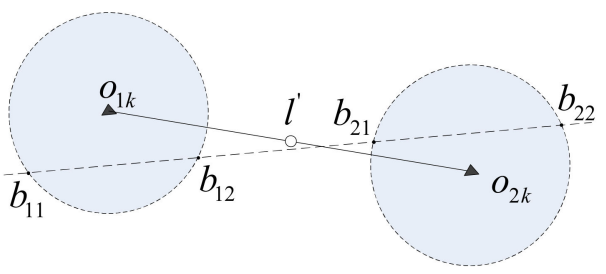

(a)

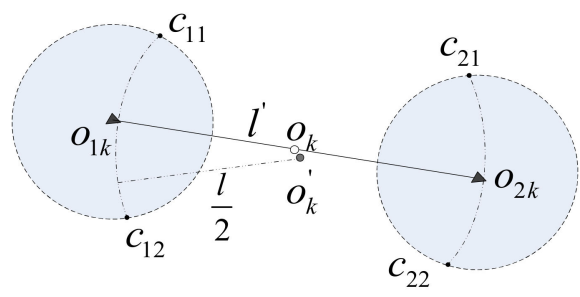

(c)

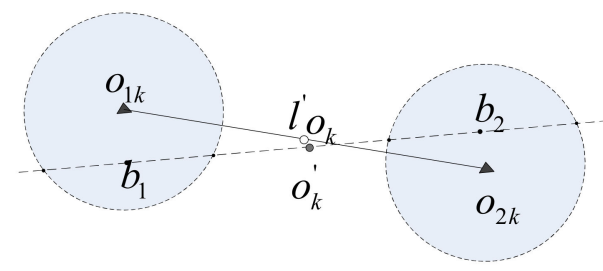

(b)

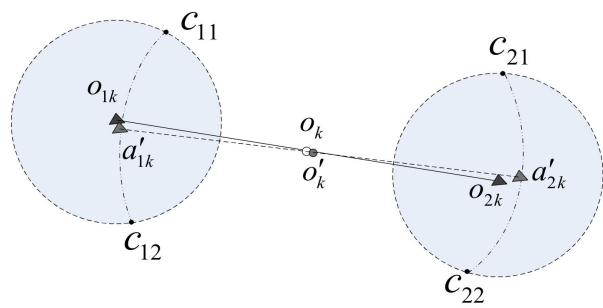

(d)

Figure 4. Scheme for joint positioning when both reader antennas detect enough tags (three at least): (a) Acquire four intersections, (b) Obtain a line $b_{1} b_{2}$, (c) Estimate the vehicle position $o_{k}^{\prime}$ and (d) Estimate the footprint centers.

\subsubsection{Only One Antenna Detects Enough Tags}

When an RFID reader antenna detects less than three RFID tags on the ground, the possible position of the virtual antenna would be in a quite large area, and thus the positioning accuracy could be very poor. For this situation, the joint-rectification positioning algorithm finds its right application.

As shown in Figure 5, suppose at time step $k$, antenna \#1 at position $a_{1 k}$ detects less than three tags so that antenna \#1 cannot be positioned. While antenna \#2 is at position $a_{2 k}$ detects more than three tags so that antenna \#2 can be positioned. $O_{k}$ is the vehicle position 
at time $k$ to be evaluated by the Monte Carlo algorithm. Since the position of antenna \#2 is more reliable, joint positioning may use the position $a_{2 k}$ to correct the position $a_{1 k}$ of antenna \#1.

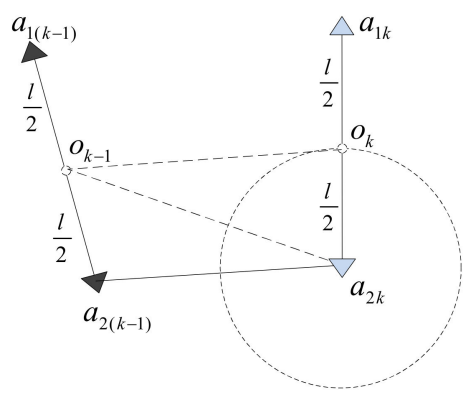

Figure 5. Scenario for positioning when only one of the antennas can detect enough tags.

Different joint positioning correction algorithms can be applied (shown in Algorithm 3). Below is a simple implementation:

(i) Use the Monte Carlo algorithm to get the position $a_{2 k}$.

(ii) Draw a circle around $a_{2 k}$, with a radius of $l / 2$.

(iii) Passing though the vehicle point $o_{k-1}$ evaluated at the time step $k-1$, draw a line tangent to the ipsilateral circle drawn in (ii). The point of tangency can be used as the position of the vehicle at time step $k$.

(iv) Connecting $a_{2 k}$ and $o_{k}$, extending the radius line to a symmetric point, which can be used as $a_{1 k}$, viz., the position of antenna $\# 1$ at time step $k$.

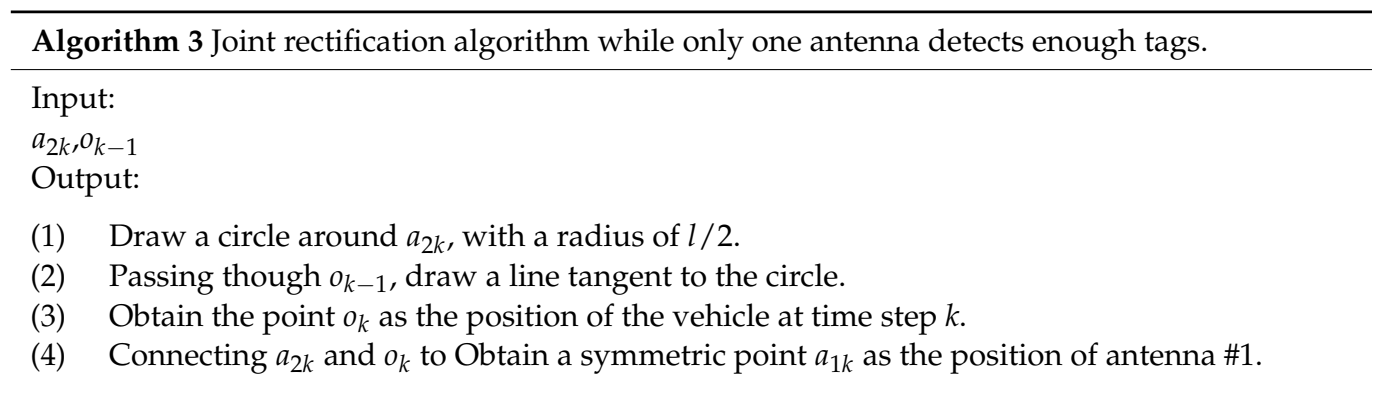

The main idea of the above correction algorithm is to estimate the location of the antenna without enough tag information by approaching the center of the sampling particles based on the distance between the two antennas.

Since the positioning in the above joint positioning scheme integrates more tag position information, the positioning accuracy can be improved for each antenna, and thus the vehicle platform. Therefore, by joint positioning with two RFID reader antennas, Monte Carlo-based RFID indoor positioning can acquire high positioning accuracy.

\section{Verification of the Monte Carlo-Based Indoor RFID Positioning}

To demonstrate the performance of the proposed Monte Carlo-based RFID indoor positioning scheme, we first verify the positioning accuracy by four numerical simulation experiments.

In the simulation, we consider a ground area of $500 \mathrm{~cm} \times 700 \mathrm{~cm}$, with 72 UHF RFID tags deployed at intervals of $50 \mathrm{~cm}$. Each reader antenna is supposed to have a footprint radius of $90 \mathrm{~cm}$ on the ground. For the Monte Carlo algorithm, we set the number of random sampling points $=300$ and the average error of odometer $=10 \%$.

The performance of positioning can be characterized by using the positioning error defined as

$$
e_{p}=\sqrt{\left(x_{k}-x_{k}^{\prime}\right)^{2}+\left(y_{k}-y_{k}^{\prime}\right)^{2}},
$$


where $x_{k}$ and $y_{k}$ are real coordinates of the vehicle at time step $k$ and $x_{k}^{\prime}$ and $y_{k}^{\prime}$ are estimated coordinates of the vehicle by the proposed positioning algorithms.

Several simulation experiments are performed on the positioning error to verify the proposed Monte Carlo-based RFID indoor positioning algorithm. In the simulation, we will set a real path for vehicle movement and calculate the positions of the vehicle with the proposed Monte Carlo-based positioning algorithm.

\subsection{Positioning of the RFID System with Two Antenna Footprints Overlapped Completely on the Ground}

When the two footprints of the RFID reader antennas overlap exactly on the ground, it gives exactly the situation of using only one antenna or one footprint as in most RFID positioning systems.

Figure 6a depicts the positioning track calculated with the Monte Carlo-based positioning algorithm. For comparison, positioning with particle filter-based method as introduced in [28] is also simulated and depicted. Figure $6 \mathrm{~b}$ depicts the positioning errors for the two positioning algorithms.

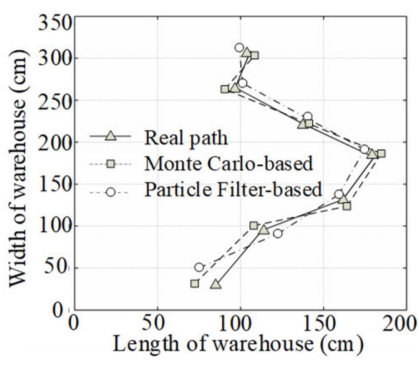

(a)

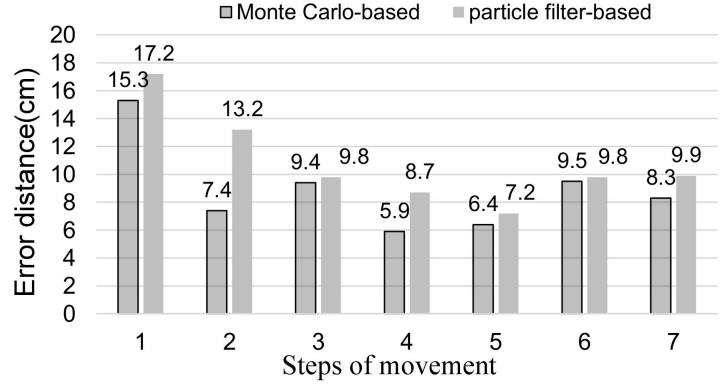

(b)

Figure 6. Simulation-based positioning results (a) and positioning errors (b) when the two antenna footprints merge into one.

From Figure 6, we find that the proposed Monte Carlo-based positioning algorithm provides an average error of approximately $8.89 \mathrm{~cm}$, while positioning with the particle filter-based algorithm provides an average error of approximately $10.83 \mathrm{~cm}$. Therefore, the Monte Carlo-based positioning may provide a better positioning accuracy even if the dual-antenna RFID positioning system uses a merged footprint.

\subsection{Positioning of RFID System with Two Antenna Footprints Separated}

When the two RFID reader antennas yield two separated footprints on the ground, the joint positioning in Section 2.3 finds its application.

Figure 7a depicts the positioning track calculated by the Monte Carlo-based positioning algorithm with the cooperation of the joint positioning for two footprints. For comparison, positioning with the particle filter-based algorithm in [28] for two antenna footprints is also simulated and depicted. Figure $7 \mathrm{~b}$ depicts the positioning errors for the two positioning algorithms.

From Figure 7, we find that the proposed Monte Carlo positioning algorithm provides an average error of approximately $5.33 \mathrm{~cm}$, while positioning with the particle filter-based algorithm provides an average error of approximately $7.51 \mathrm{~cm}$. The proposed Monte Carlo-based positioning still provides a higher positioning accuracy.

Compared to the Monte Carlo-based positioning algorithm for one footprint shown in Figure 6, the proposed Monte Carlo-based positioning for two footprints improves the positioning accuracy significantly, from an average error of approximately $8.89 \mathrm{~cm}$ to $5.33 \mathrm{~cm}$. 


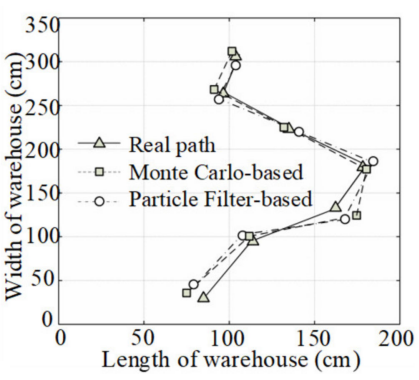

(a)

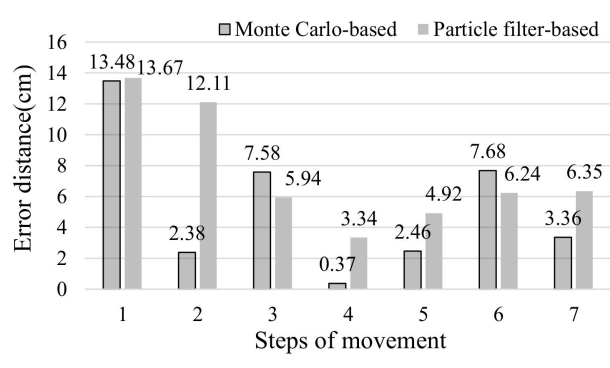

(b)

Figure 7. Simulation-based positioning results (a) and positioning errors (b) when the dual-antenna RFID system has two separated footprints.

It is interesting to see the comparison to the positioning with the particle filter-based algorithm in [28] for a single but large footprint, viz., with a footprint radius of $128 \mathrm{~cm}$, which gives an area equal to the sum of two antenna footprints. Figure 8a depicts the positioning track calculated with Monte Carlo-based positioning algorithm for two footprints and with the particle filter-based algorithm for one large footprint. Figure $8 \mathrm{~b}$ depicts the positioning errors for the two positioning algorithms.

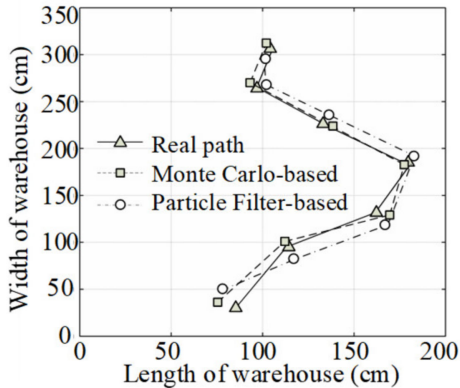

(a)

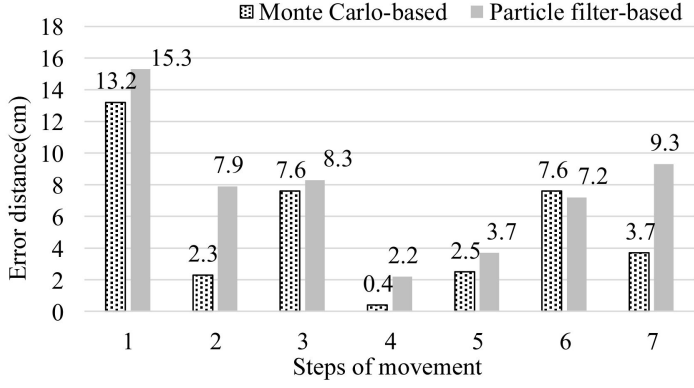

(b)

Figure 8. Simulation-based positioning results (a) and positioning errors (b) when the particle filter-based algorithm uses one large footprint with a radius of $128 \mathrm{~cm}$.

From Figure 8, we find that the positioning with the particle filter-based algorithm using one large footprint provides an average error of approximately $7.70 \mathrm{~cm}$, a bit worse than the positioning with the particle filter-based algorithm using two footprints.

With the above simulation experiments, the performance of the proposed Monte Carlo-based RFID positioning scheme is demonstrated. Better positioning accuracy can be expected for the Monte Carlo-based positioning.

\subsection{Experimental Results}

Three test experiments have been carried out to demonstrate the performance of the Monte Carlo-based RFID positioning scheme. The experiment system is shown in Figure 9. In the system, a laptop computer serves as the intelligent control center of the moving platform. On the platform, an Alien UHF RFID reader ALR 9900 with polling for ts is applied. Additionally, two output ports of the reader are connected to two circulaly polarized microstrip patch antennas, of which the central operating frequency is $915 \mathrm{M}$ $\mathrm{Hz}$, and the orientation can be adjusted flexibly. On the floor, 72 passive RFID tags are deployed in an area of $500 \mathrm{~cm} \times 700 \mathrm{~cm}$, as defined in the simulation experiment. With the ID and position information of all tags on the floor, an electronic map can be formed in the intelligent control center. 


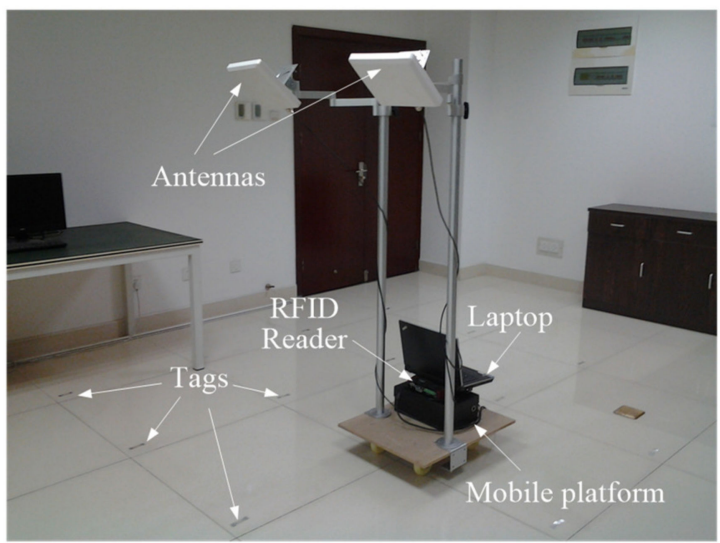

Figure 9. The indoor positioning system.

To perform experiments as in the simulations, we carefully adjust the output power of Alien ALR 9900 and antenna height, so that footprint of a radius of approximately $90 \mathrm{~cm}$ is acquired.

To measure the footprint on the ground, we fix the platform and thus the RFID reader antenna, move an RFID tag on the floor, and record the boundary of the RFID tag that it gives a response to the reader's interrogation.

In the experiments, overlapped or separated footprints can be acquired by tilting the two antennas properly so that the beam direction can be adjusted, as shown in Figure 2. Three experiments with different footprints on the ground have been carried out to demonstrate the performance of the proposed Monte Carlo-based RFID positioning scheme.

\section{Experimental Results}

\subsection{Positioning of RFID System with Two Antenna Footprints Overlapped Completely on} the Ground

By adjusting the height and tilt angles of the two reader antennas in Figure 9, the two footprints on the floor can overlap on the floor, which gives a single footprint of a radius of $90 \mathrm{~cm}$.

Figure 10a shows the positioning track calculated by using the test data. For comparison, positioning by using both the proposed Monte Carlo-based algorithm and the particle filter-based algorithm in [28] are depicted. Figure 10b shows the average positioning errors. For the Monte Carlo-based positioning algorithm, it is $9.72 \mathrm{~cm}$, while for the particle filter-based algorithm, it is $17.18 \mathrm{~cm}$. Therefore, the Monte Carlo-based positioning yields higher positioning accuracy.

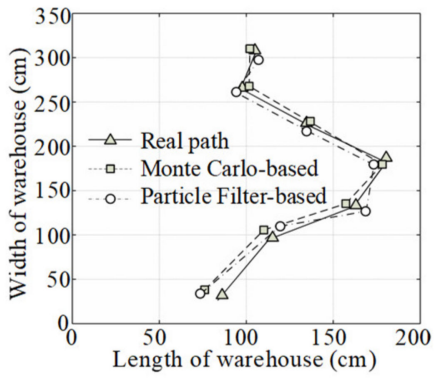

(a)

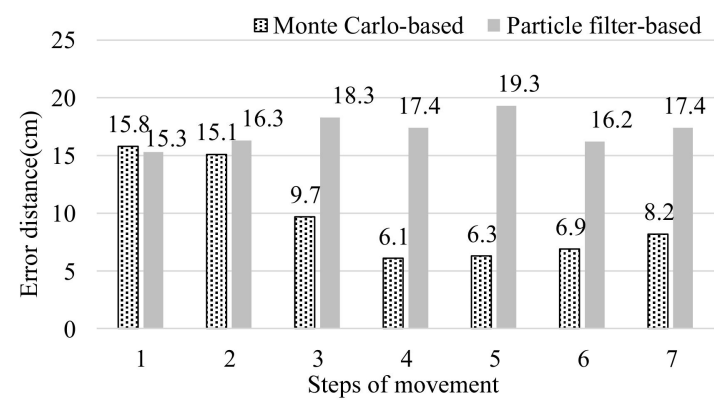

(b)

Figure 10. Experimental positioning results (a) and positioning errors (b) when the two antenna footprints merge into one. 


\subsection{Positioning of RFID System with Two Antenna Footprints Separated}

By adjusting the height and tilt angles of the two reader antennas in Figure 9, two separated footprints with a radius of $90 \mathrm{~cm}$ can be generated on the floor.

Figure 11a depicts the positioning track calculated by the proposed Monte Carlo-based positioning algorithm with the cooperation of the joint positioning for the two footprints. For comparison, positioning with the particle filter-based algorithm in [28] for two antenna footprints is also tested and depicted.

From Figure 11a, we find that both the two positioning algorithms give a satisfactory positioning. From Figure 11b, we may calculate the average positioning error. For the proposed Monte Carlo-based positioning algorithm, it is $8.87 \mathrm{~cm}$, while for the particle filter-based algorithm, it is $13.42 \mathrm{~cm}$. Therefore, the proposed Monte Carlo-based positioning yields higher accuracy in practice.

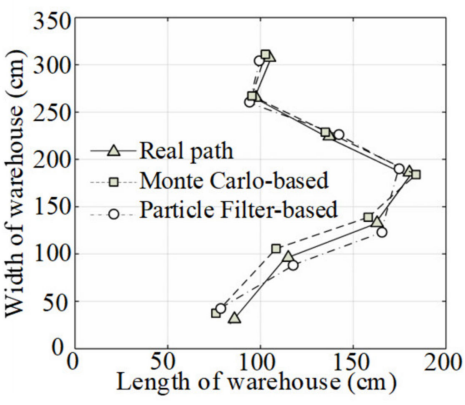

(a)

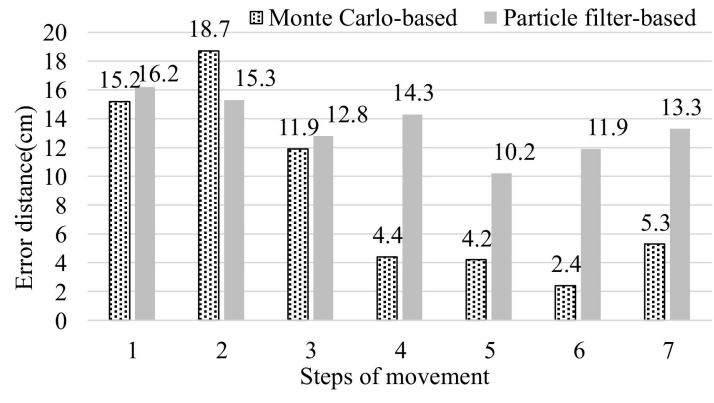

(b)

Figure 11. Experimental positioning results (a) and positioning errors (b) when the dual-antenna RFID positioning system has two separated footprints.

\subsection{Comparison to the Positioning of an RFID System with One Large Footprint}

By connecting just one antenna to the ALR 9900 and adjusting the height and tilt angles of the antennas in Figure 9, one large footprint with a radius of $128 \mathrm{~cm}$ can be generated on the floor.

Positioning with the particle filter-based algorithm in [28] for the large antenna footprints is tested. Figure 12a depicts the positioning result. For comparison, positioning with the proposed Monte Carlo-based algorithm for two antenna footprints, as shown in Figure 11a, is also depicted. Figure $12 \mathrm{~b}$ depicts the positioning errors for the two positioning algorithms.

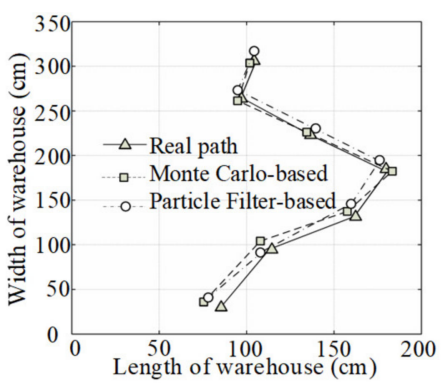

(a)

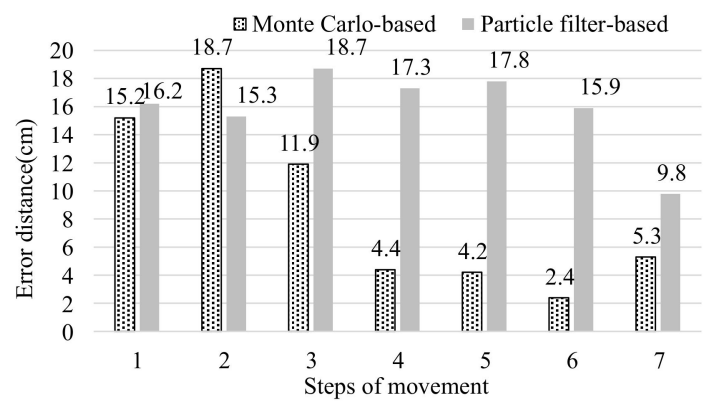

(b)

Figure 12. Experiment positioning results (a) and positioning errors (b) when the particle filter-based algorithm uses one large footprint of a radius of $128 \mathrm{~cm}$.

From Figure 12b, we may calculate the average positioning error. For the positioning with the particle filter-based algorithm in [28], it is $15.86 \mathrm{~cm}$. The proposed Monte Carlobased positioning algorithm yields higher accuracy in practice. 
It should be noted that the experiment positioning results are worse than those acquired based on the simulation. The reason is that in the experiments, the polarization mismatch between the reference passive RFID tags and the reader antennas can not be avoided, while in the simulation, there is no polarization mismatch.

The above three positioning tests demonstrate that the proposed Monte Carlo algorithm with the cooperation of the joint positioning can provide higher accuracy in practice.

A comparison of the indoor positioning accuracy with different typical positioning schemes is listed in Table 1. It is found that the proposed Monte Carlo-based dual-antenna scheme may achieve satisfactory accuracy for the self-positioning of a moving vehicle.

Table 1. Indoor positioning accuracy with different typical positioning schemes.

\begin{tabular}{cc}
\hline Positioning Scheme & Mean Positioning Error \\
\hline WiFi-based [2] & $<0.2 \mathrm{~m}$ \\
Bluetooth-based [3] & $<1.43 \mathrm{~m}$ \\
Visible light-based [4] & $<0.02 \mathrm{~cm}$ \\
RFID-based, presented in this paper & $<0.1 \mathrm{~m}$ \\
\hline
\end{tabular}

\section{Further Discussion}

Although Monte Carlo-based RFID positioning yields high positioning precision, there are still several practical factors that may affect its performance. Detailed discussion about such effects will benefit the practical deployment of the Monte Carlo-based RFID positioning system. The following discussion will be majorly based on simulation. In the simulation, we use the same simulation setup as the system with two footprints depicted in Section 3.

\subsection{Selection of Number of Random Sampling Particle}

The number of random sampling particles in the Monte Carlo-based positioning algorithm determines how many sampling particles are required to satisfy the system condition in a footprint. Thus, it will affect the positioning error. On the other hand, using too many random sampling particles will decrease the system's efficiency.

Figure 13 depicts the effects of the number of sampling particles $N_{0}$ on the average positioning error. From Figure 13, we find that for $N_{0} \geq 200$, there is no significant difference in the average error. However, the selection of a too-large number usually requires more computation time for positioning, which will degrade the real-time performance of positioning.

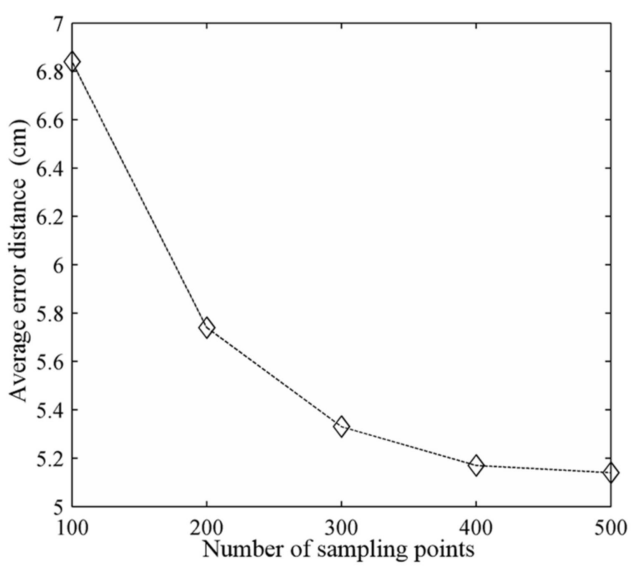

Figure 13. Effects of sampling particle number on the average positioning errors.

\subsection{Density of RFID Tag on the Ground}

From the analysis in Section 2, it is known that the positioning accuracy will degrade 
when there are not enough RFID tags in the footprint on the floor. For a specified footprint on the floor and designated sampling particle number $N$, there should be an optimal tag density on the floor. A too-dense tag distribution will increase the cost of an RFIDbased positioning system, while a too-sparse tag distribution will not guarantee necessary positioning accuracy.

For $N_{0}=200$, Figure 14 shows the effects of tag density on the average positioning error for a footprint of a radius of $90 \mathrm{~cm}$.

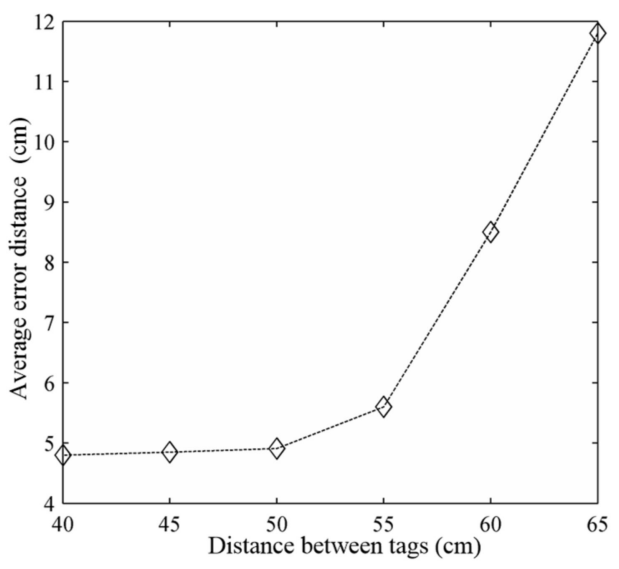

Figure 14. Effects of tag interval on the average positioning error.

From Figure 14, it is observed that when the interval between two RFID tags on the floor is larger than $50 \mathrm{~cm}$, the average positioning error increases drastically. The reason is that for a footprint of a radius of $90 \mathrm{~cm}$, the number of tags detected by each antenna may not be sufficient. The sparser the reference tags on the floor, the larger the sampling area and the more the sampling particles.

It should be noted that, since the sampling number also plays an important role in acquiring desired average positioning error, the sampling number should also be included in determining the tag density on the floor. Figure 15 depicts the joint influence of tag density and sampling number on average positioning error.

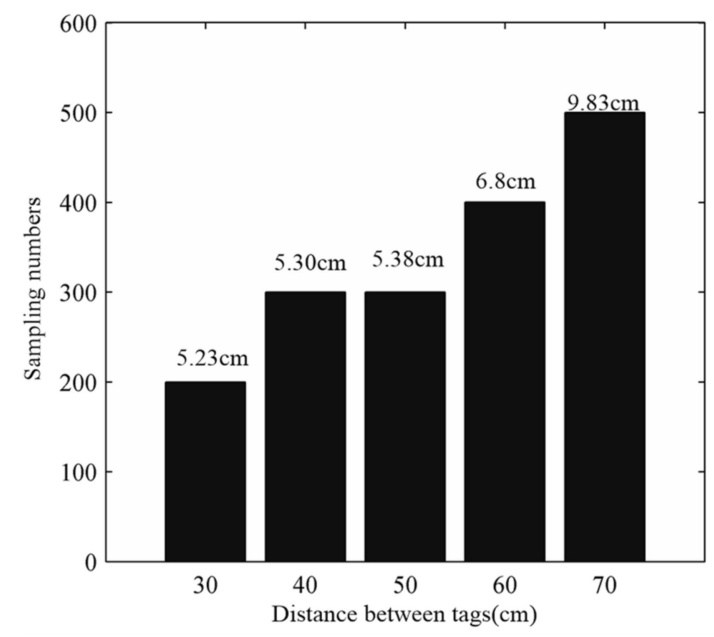

Figure 15. Effects of tag density and sampling number on the average positioning error.

From Figure 15, we have the observation that a too-dense tag distribution on the floor is not necessary because we do not find significant improvement in positioning accuracy. For a sparse tag distribution on the floor, increasing the sampling number may benefit the positioning accuracy. However, the tag density gives a decisive effect. 


\subsection{RFID Reader System}

There are several factors that may affect the positioning accuracy in the RFID system, such as the footprint size, the distance between the two antennas, and the polling time interval of the two antennas' interrogation. Effects of the footprint size and footprint overlap are illustrated as follows.

\subsubsection{Influence of the Footprint Size}

Figure 16 shows the relationship between the positioning accuracy and the footprint size of each antenna. In the calculation, we set the other parameters of the RFID system as in Section 3 except the footprint radius.

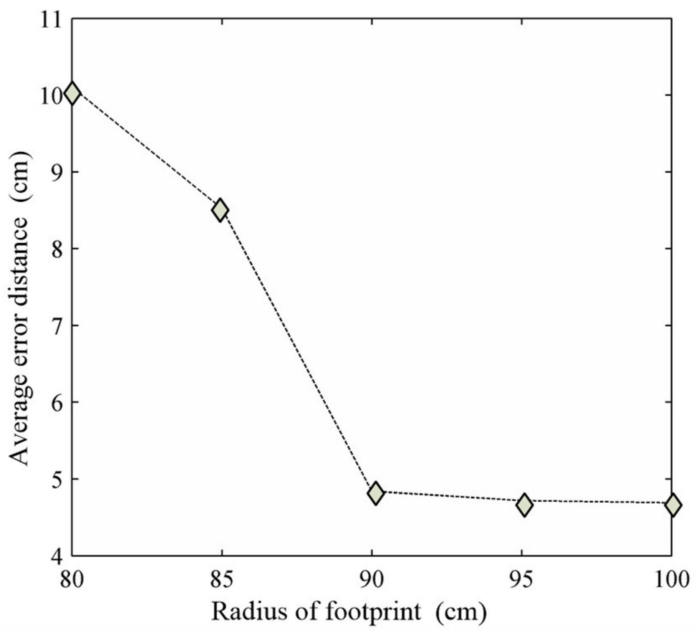

Figure 16. Effects of footprint radius on the average positioning error.

It is found in Figure 16 that for $R<90 \mathrm{~cm}$, the positioning accuracy decreases severely because the footprint is too small to interrogate enough tags for positioning reference. For $R \geq 90 \mathrm{~cm}$, enough reference tags can be interrogated so that the errors converge to a satisfactory value. For practical situations, different deployments of reference RFID tags may have different footprint size requirements.

\subsubsection{Influence of the Footprint Overlap}

The footprint overlap will also affect the positioning accuracy. The reason is that a tag different parts of the common area of the two footprints may have different detectability for different antenna channels, which is especially the case in practical situations.

The detectability of a tag in a footprint can be defined by using a certain probability model. Overall, the tag at a position far from the footprint center has low detectability. For the dual-antenna RFID system, it is reasonable to use a simple probability model as

$$
p(l)=\lambda \cdot e^{-l},
$$

where $\lambda$ is a parameter related to the indoor environment and $l$ is the distance between the two antennas. The relationship between antenna distance and the overlap extent of antenna footprint can be derived according to the geometry shown in Figure 2.

Figure 17 depicts the effects of footprint overlap, in terms of the antenna distance, on the positioning accuracy. To indicate the effects of antenna distance at different frequencies, distance normalized to the wavelength at the center frequency of UHF RFID is applied. In the simulation, we set the other parameters of the RFID system as in Section 3 except the introduction of the detectability probability as defined in (11). 


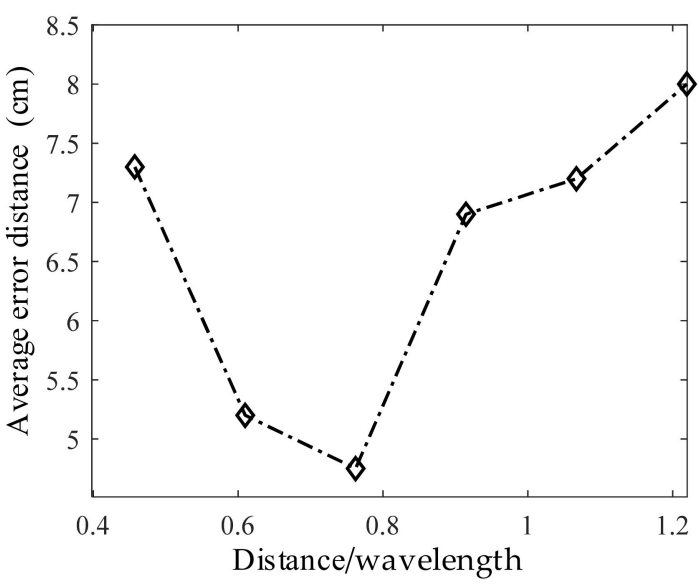

Figure 17. Effects of antenna distance on the average positioning error.

From Figure 17, it is found that there is an optimal distance between the two antennas. For the experiment system operating in the lab, as shown in Figure 9, it is around the 0.76 wavelength.

For different dual-antenna RFID positioning systems and in different indoor environments, the optimal antenna distance should be carefully adjusted.

Therefore, the performance of the proposed scheme would be influenced by the number of sampling particle, tags interval, footprint radius, and antenna distance. Other occurrences in practice, such as the invalidation of certain reference tags due to damage or the misinformation of certain reference tags due to vicinity interrogation from other readers, can be equivalent to a change to the tag interval or footprint radius at the stage that the moving vehicle was passing through the occurrence area. Since the Monte Carlo-based positioning is a step-by-step scheme, the influence of such occurrence can be rectified by the following accumulative positioning. This can be understood by comparing the positioning errors in Figures $10 \mathrm{~b}, 11 \mathrm{~b}$ and $12 \mathrm{~b}$. At the start, the positioning errors are always a bit larger because there is not enough tag information (viz., equivalent to certain disturbing occurrence), and then the accuracy is improved when there is enough tag information (viz., after the vehicle is rid of the disturbing occurrence).

\section{Conclusions}

For moving platforms carrying an RFID system with two antennas, a novel RFIDbased indoor self-positioning scheme is proposed by using a Monte Carlo-based positioning algorithm with the cooperation of dual-antenna rectification.

By deploying reference passive RFID tags with known location information on the ground, an RFID tag-based map can be formed and loaded into the platform system. Indoor self-positioning of the moving platform carrying an RFID reader with two forward-looking antennas can be implemented, in a very straightforward manner, by looking up the positions of all responded RFID tags at each time step of movement, and estimating the platform position by using the proposed Monte Carlo-based algorithm.

To improve the positioning accuracy of Monte Carlo-based positioning, each antenna channel, with its own footprint on the ground, may rectify its position estimation by using the tag position information interrogated by the other antenna channel. Both simulation and experiment tests demonstrate there is a positioning accuracy improvement compared with the particle filter-based algorithm in [28]. The experiment results indicate that the proposed algorithm can achieve a minimum positioning error of $8.87 \mathrm{~cm}$, which is $33.9 \%$ lower than the particle filter-based algorithm. For practical deployment of the proposed RFID-based self-positioning system, some factors in a practical indoor-positioning system, such as the reference tag distribution pattern, reader antenna footprint size, and footprint overlap, should be designed. 
In the future, reference passive RFID tags on indoor objects and facilities, not on the ground but with known location information, can be included to improve further the positioning precision. For applications in a much complex environment, optimization techniques for the deployment of reference tags will be developed to improve further the positioning accuracy of the proposed RFID positioning technique.

Author Contributions: Conceptualization, G.W. and J.S.; investigation, L.W. and Y.T.; software, L.W.; validation, L.W. and Y.T.; data curation, Y.T.; writing-original draft preparation, Y.T.; writingreview and editing, G.W. and J.S.; funding acquisition, Y.T. and G.W. All authors have read and agreed to the published version of the manuscript.

Funding: Natural science fund for colleges and universities in Jiangsu Province (Grant No. 20KJB5100 05), Scientific Research Foundation for Doctors of Jinling Institute of Technology (Grant No. jit-b201718), and the Key Research Program of Frontier Sciences of CAS under Grant QYZDY-SSW-JSC035.

Conflicts of Interest: The authors declare no conflict of interest.

\section{References}

1. Huang, B.; Xu, Z.; Jia, B.; Mao, G. An online radio map update scheme for WiFi fingerprint-based localization. IEEE Internet Things J. 2019, 6, 6909-6918. [CrossRef]

2. Zhao, Y.; Xu, J.; Wu, J.; Hao, J.; Qian, H. Enhancing camera-based multimodal indoor localization with device-free movement measurement using WiFi. IEEE Internet Things J. 2020, 7, 1024-1038. [CrossRef]

3. Al-Madani, B.; Orujov, F.; Maskeliūnas, R.; Damaševičius, R.; Venčkauskas, A. Fuzzy logic type-2 based wireless indoor localization system for navigation of visually impaired people in buildings. Sensors 2019, 19, 2114. [CrossRef]

4. Naz, A.; Asif, H.M.; Umer, T.; Kim, B. PDOA based indoor positioning using visible light communication. IEEE Access 2018, 6, 7557-7564. [CrossRef]

5. Yamano, K.; Tanaka, K.; Hirayama, M.; Kondo, E.; Kimura, Y.; Matsumoto, M. Self-localization of mobile robots with RFID system by using support vector machine. Proc. IEEE Int. Conf. Intell. Robot. Syst. 2004, 4, 3756-3761.

6. Medeiros, C.; Costa, J.; Fernandes, C. RFID reader antennas for tag detection in self-confined volumes at UHF. IEEE Antennas Propag. Mag. 2011, 53, 39-50. [CrossRef]

7. Choi, B.; Lee, J.; Lee, J.; Park, K. A hierarchical algorithm for indoor mobile robot localization using RFID sensor fusion. IEEE Trans. Ind. Electron. 2011, 58, 2226-2235. [CrossRef]

8. Han, S.; Lim, H.; Lee, J. An efficient localization scheme for a differential-driving mobile robot based on RFID system. IEEE Trans. Ind. Electron. 2007, 54, 3362-3369. [CrossRef]

9. Milella, A.; Cicirelli, G.; Distante, A. RFID-assisted mobile robot system for mapping and surveillance of indoor environments. Ind. Robot Int. J. 2008, 35, 143-152. [CrossRef]

10. Scherhäufl, M.; Pichler, M.; Schimbäck, E.; Müller, D.J.; Ziroff, A.; Stelzer, A. Indoor localization of passive UHF RFID tags based on phase-of-arrival evaluation. IEEE Trans. Microw. Theory Tech. 2013, 61, 4724-4729. [CrossRef]

11. Cremer, M.; Dettmar, U.; Hudasch, C.; Kronberger, R.; Lerche, R.; Pervez, A. Localization of passive UHF RFID tags using the AoAct transmitter beamforming technique. IEEE Sens. J. 2016, 16, 1762-1771. [CrossRef]

12. Subedi, S.; Pauls, E.; Zhang, Y.D. Accurate localization and tracking of a passive RFID reader based on RSSI measurements. IEEE J. Radio Freq. Identif. 2017, 1, 144-152. [CrossRef]

13. Scherhäufl, M.; Rudić, B.; Stelzer, A.; Pichler-Scheder, M. A blind calibration method for Phase-of-Arrival-Based localization of passive UHF RFID transponders. IEEE Trans. Instrum. Meas. 2019, 68, 261-268. [CrossRef]

14. Subramanian, S.P.; Sommer, J.; Schmitt, S.; Rosenstiel, W. RIL-Reliable RFID based indoor localization for pedestrians. In Proceedings of the 16th International Conference on Software, Telecommunications and Computer Networks, Split, Croatia, 25-27 September 2008; pp. 218-222.

15. Saad, S.S.; Nakad, Z.S. A standalone RFID indoor positioning system using passive tags. IEEE Trans. Ind. Electron. 2011, 58, 1961-1970.

16. Ruiz, A.R.J.; Granja, F.S.; Honorato, J.C.P.; Rosas, J.I.G. Accurate pedestrian indoor navigation by tightly coupling foot-mounted IMU and RFID measurements. IEEE Trans. Instrum. Meas. 2012, 61, 178-189. [CrossRef]

17. Song, X.; Li, X.; Tang, W.; Zhang, W.; Li, B. A hybrid positioning strategy for vehicles in a tunnel based on RFID and in-vehicle sensors. Sensors 2014, 14, 23095-23118. [CrossRef] [PubMed]

18. Huang, C.-H.; Lee, L.-H.; Ho, C.C.; Wu, L.-L.; Lai, Z.-H. Real-time RFID indoor positioning system based on Kalman-filter drift removal and Heron-bilateration location estimation. IEEE Trans. Instrum. Meas. 2015, 64, 728-739. [CrossRef]

19. Ma, H.; Wang, K. Fusion of RSS and phase shift using the Kalman filter for RFID tracking. IEEE Sens. J. 2017, 17, 3551-3558. [CrossRef]

20. Zhao, Y.; Liu, K.; Ma, Y.; Gao, Z.; Zang, Y.; Teng, J. Similarity analysis-based indoor localization algorithm with backscatter information of passive UHF RFID tags. IEEE Sens. J. 2017, 17, 185-193. [CrossRef] 
21. DiGiampaolo, E.; Martinelli, F. Mobile robot localization using the phase of passive UHF-RFID signals. IEEE Trans. Ind. Electron. 2014, 61, 365-376. [CrossRef]

22. Fan, Q.; Zhang, H.; Sun, Y.; Zhu, Y.; Zhuang, X.; Jia, J.; Zhang, P. An optimal Enhanced Kalman filter for a ZUPT-Aided pedestrian positioning coupling model. Sensors 2018, 18, 1404. [CrossRef] [PubMed]

23. Athalye, A.; Savić, V.; Bolić, M.; Djurić, P.M. Radio frequency identification system for accurate indoor localization. In Proceedings of the IEEE International Conference on Acoustics, Speech and Signal Processing (ICASSP), Prague, Czech Republic, 22-27 May 2011; pp. 1777-1780.

24. Nemmaluri, A.; Corner, M.D.; Shenoy, P. Sherlock: Automatically locating objects for humans. In Proceedings of the 6th International Conference on Mobile Systems, Applications, and Services, Breckenridge, CO, USA, 17-20 June 2008; pp. 187-198.

25. Kim, M.; Chong, N.; Ahn, H.; Yu, W. RFID-enabled target tracking and following with a mobile robot using direction finding antennas. In Proceedings of the IEEE International Conference on Automation Science and Engineering, Scottsdale, AZ, USA, 22-25 September 2007; pp. 1014-1019.

26. Deyle, T.; Kemp, C.C.; Reynolds, M.S. Probabilistic UHF RFID tag pose estimation with multiple antennas and a multipath RF propagation model. In Proceedings of the IEEE/RSJ International Conference on Intelligent Robots and Systems, Nice, France, 22-26 September 2008; pp. 1379-1384.

27. Jia, S.; Sheng, J.; Takase, K. Improvement of performance of localization ID tag using multi-antenna RFID system. In Proceedings of the SICE Annual Conference, Chofu, Japan, 20-22 August 2008; pp. 1715-1718.

28. Park, S.; Lee, H. Self-recognition of vehicle position using UHF passive RFID tags. IEEE Trans. Ind. Electron. 2013, 60, 226-234. [CrossRef]

29. Aguilar-Garcia, A.; Fortes, S.; Barco, R.; Colin, E. Enhancing localization accuracy with multi-antenna UHF RFID fingerprinting. In Proceedings of the IEEE International Conference on Indoor Positioning \& Indoor Navigation, Banff, AB, Canada, 13-16 October 2015.

30. Ma, Y.; Wang, B.; Pei, S.; Zhang, Y.; Zhang, S.; Yu, J. An indoor localization method based on AOA and PDOA using virtual stations in multipath and NLOS environments for passive UHF RFID. IEEE Access 2018, 6, 31772-31782. [CrossRef]

31. Dellaert, F.; Fox, D.; Burgard, W.; Thrun, S. Monte Carlo localization for mobile robots. In Proceedings of the IEEE International Conference on Robotics and Automation (ICRA99), Detroit, MI, USA, 10-15 May 1999.

32. Doucet, A.; Gordon, N.J.; Krishnamurthy, V. Particle filters for state estimation of jump Markov linear systems. IEEE Trans. Signal Process. 2001, 49, 613-624. [CrossRef]

33. Zhang, H.; Chen, J.C.; Zhang, K. RFID-based localization system for mobile robot with Markov Chain Monte Carlo. In Proceedings of the 2014 Zone 1 Conference of the American Society for Engineering Education (ASEE Zone 1), Bridgeport, CT, USA, 3-5 April 2014.

34. Wang, J.; Bolic, M. Accurate localization using augmented UHF RFID system for Internet-of-Things. In Proceedings of the Thirteenth International Conference on Wireless and Mobile Communications (ICWMC 2017), Nice, France, $23-27$ July 2017. 